\title{
Review on Pharmacological Activity of Amygdalin
}

\author{
Muhammad Qadir* and Kiran Fatima
}

Institute of Molecular Biology and Biotechnology, Bahauddin Zakariya University, Multan, Pakistan

${ }^{*}$ Corresponding author: Muhammad Imran Qadir, Institute of Molecular Biology and Biotechnology, Bahauddin Zakariya University, Multan, Pakistan. Tel: +92-301-2725746; E-mail mrimranqadir@hotmail.com

Received: October 14, 2017; Accepted: November 06, 2017; Published: November 08, 2017

Citation: Qadir M, Fatima K (2017) Review on Pharmacological Activity of Amygdalin. Arch Can Res Vol.5:No.4:160.

\section{Abstract}

Amygdalin is obtained from the pebbles of rosaceous fruits, like apricots, almond, cherries, peaches and plums. It is a plant glucoside which is traditionally used as antitumor drug. It produces synergistic effect if it combines with conditional chemotherapy drugs. Amygdalin also used to cure many other diseases like to control asthma, improve immune system, causes apoptosis of human renal fibroblast, inhibit hyperglycemia. Amygdalin is banning to use as antitumor drug by FDA due to lack evidences of cure in case of cancer but in ancient times it is used as antitumor drug by Chinese.

Keywords: Amygdalin; Anti-tumor; Toxicity; Antiinflammatory; Analgesics

\section{Introduction}

Amygdalin which is also called laetrile is the aromatic cyanogenic compound. Amygdalin is a plant glucoside which belongs to Rosaceae family. It is also known as vitamin B17 easily isolated from apricot stones, almonds, cherries, peaches and plums. Its chemical formula is $\mathrm{C}_{20} \mathrm{H}_{10} \mathrm{NO}_{11}$ and molecular weight of this cyanogenic compound is 457.42 Dalton. Dmandelonitrile- $\beta$-D-glucoside- $6-\beta$-glucoside is the chemical structure of laetrile. Amygdalin is outdated drug has various advantages like antipyretic, antitussive, anticancer and thirstquenching effects. In ancient days it is used to treat numerous infections like asthma, nausea, leprosy, bronchitis and leukoderma [1]. Amygdalin itself is nontoxic but it produces poisonous substance HCN which is decomposed by enzymes. The pharmacological effects of laetrile also comprise antiatherogenic, shyness of renal fibrosis, avoidance of pulmonary fibrosis, fight to hyperoxia brought lung injury, immune regulation, antitumor, anti-inflammatory [2]. Antitumor effect of amygdalin is one of the newest topic in recent years. The anticancer function of laetrile is regulated by carcinogenic substances which is decomposed in the body and destroy tumor cells, blocking nutrients of cancerous cells, prevents tumor cell growth and decrease growth of prostate cancer. It has been manufactured and used in 20 countries for cure of cancer by traditional method like in Italy, Japan, China and America. Although it was encouraged as cancer cure but when it is clinically inspected it is not permitted by FDA due to inadequate clinical proofs and its low potential and effectiveness to kill tumor or modified cells.

\section{Literature Review}

\section{Anti-asthmatic effect}

Amygdalin can promote synthesis of pulmonary surfactant. Amygdalin is decomposed into benzaldehyde and hydrocyanic acid after oral administration which prevent respiratory center to reach certain level and slow down respiratory movement and produce antitussive and antiasthmatic effect. Amygdalin or laetrile used for prevention of asthma in Korean traditional medicine. However, proper antiasthmatic action of amygdalin still not completely understood. Amygdalin do not engulf or kill the type 1 helper T cell response, but it kills the type 2 helper $T$ cell. Route of Oral administration of amygdalin weakens asthmatic appearances including AHR and airway infection, which result in the case of inhibition of Th2 response to allergen [3].

\section{Enhanced caspase-mediated cell death of human renal fibroblast}

Laetrile can promote action of type 1 collagenase released by human kidney fibroblast in definite action of time and concentration which inhibit certain expression of type 1 collagenase and human kidney fibroblast production, which encourage apoptosis of human renal fibroblast [4,5].

\section{Role in immune system}

Laetrile can promote polyhydroxyalkanoates induced human $\mathrm{T}$ lymphocytes proliferation and increase blood lymphocytes motivated by polyhydroxyalkanoates which secret IL-2 and Interferon and prevent TGF- $\beta 1$ thus enhancing immune response. Due to the stimulation of regulatory $T$ Cells Amygdalin has anti-atherosclerotic effect on Apo lipoprotein $E$ deficient (ApoE-/-) mice. Amygdalin functions depends upon the TGF- $\beta$ manner, and encourage apoptosis or caspase mediated cell death in plaque area. Amygdalin has the therapeutic potential of treating and preventing of atherosclerosis $[4,5]$. 


\section{Pain relieving effect}

It is confirmed that amygdalin has pain relieving effects after test on mouse hot plate which is induce by acetic acid. Pain in rats is less by amygdalin obtained from Prunus armeniaca because it produces less formalin content. The mechanism involves with inflammatory cytokines such as TNF and IL-1 moreover amygdalin produce anti-inflammatory an analgesic effect by inhibiting prostaglandin and nitric oxide production by defeating lipopolysaccharide encouraged cyclooxygenase-2 [6]. The mechanism of antinociceptive activity of amygdalin is reported by some research reports. Benzaldehyde unit of amygdalin chemical structure play important part in antinociceptive action. The anti-inflammatory and antinociceptive effects of amygdalin in mouse BV2 microglial cells was recently reported. Evidence has been gathered both in vitro and in animal models pointing to the antiinflammatory and analgesic characteristics of amygdalin, through inhibition of pro-inflammatory cytokines release, as well as TNF-a, IL-1b or IL-6, or other mediators like the c-Fos protein, cyclooxygenase or nitric oxide synthase [7].

\section{Effect on digestive system}

When the route of amygdalin administration is oral then it is decomposed into prunasin by enzymes present in digestive tract after passing through the salivary and gastrointestinal stages. The major component of amygdalin in digestive fluids is prunasin which was incubated in a caco-2 cell culture system. prunasin was decomposed into the mandelonitrile by $\beta$-glucosidase and hydroxylase through the small intestinal wall producing hydroxymandelonitrile. Benzaldehyde is also component of amygdalin when it is decomposed by enzymes it can reduce pepsin activity and cause damage to digestive function. The dose of $500 \mathrm{mg} / \mathrm{kg}$ of pepsin hydrolysate obtained from almond water on $\mathrm{CCl}_{4}$ is given to rats which establish it inhibits the level of AST, ALT and rise hydroxyproline content, inhibit extension of euglobulin lysis time. The almond water can stop the multiplication of connective tissue of rat liver and induced the increase of rat's ALT, AST level [8-10].

\section{Inhibit hyperglycemia}

Amygdalin prevents the alloxan induced hyperglycemia which depends on effective concentration of drug in blood. Research has shown therapeutic effect of amygdalin for gastric ulcer. It also induces angiogenesis in the diabetic rats. Amygdalin is given to male Swiss-Webster mice at $3 \mathrm{~g} / \mathrm{kg}$ I hour prior to $75 \mathrm{mg} / \mathrm{kg}$ against the diabetogenic actions of alloxan. The protection given by amygdalin is due to its scavenging of the harmful and highly sensitive hydroxyl radical which was produced from alloxan $[9,10]$.

\section{Toxicity of amygdalin}

The toxicity of amygdalin through intravenous route is less good than the toxicity of oral administration. In rats the fatal dose $\left(L_{50}\right)$ of oral administration of amygdalin is described as $880 \mathrm{mg} / \mathrm{kg}$ body weight in rats. In case of intravenous injection in rats the lethal dose $\left(\mathrm{LD}_{50}\right)$ of amygdalin are $25 \mathrm{~g} / \mathrm{kg}$. In case of intraperitoneal injection, the $L_{50}$ or fatal dose of amygdalin are $8 \mathrm{~g} / \mathrm{kg}$. Human lethal dose of intravenous injection is $5 \mathrm{~g}$. After oral administration amygdalin is produce more hydrocyanic acid as it is hydrolyzed or decomposed by intestinal microbes. Toxicity in human when amygdalin is orally administrated is $4 \mathrm{~g}$ per day for 15 days is shown and intravenous injection for month. If dose is reduced to daily oral doses, then it can avoid toxicity [10].

\section{Chemistry of amygdalin}

Laetrile is a cyanogenic glycoside obtain from the aromatic amino acid phenylalanine. It is obtained from stones of fruit such as apricot (8\%) bitter almond (5\%) and plum (2. 5\%). Stones are taken out from fruit and dried in sunlight or oven. Then kernels are boiled in ethanol after evaporation add diethyl ether amygdalin is precipitated in white crystals. Laetrile is degraded by intestinal $\beta$-glucosidase, suspension and amygdales to gentiobiose and L-mandelonitrile. Gentiobiose is further hydrolyze to glucose while Lmandelonitrile in hydrogen cyanide and benzaldehyde. Hydrogen cyanide in sufficient amount cause poisoning (Figure 1).

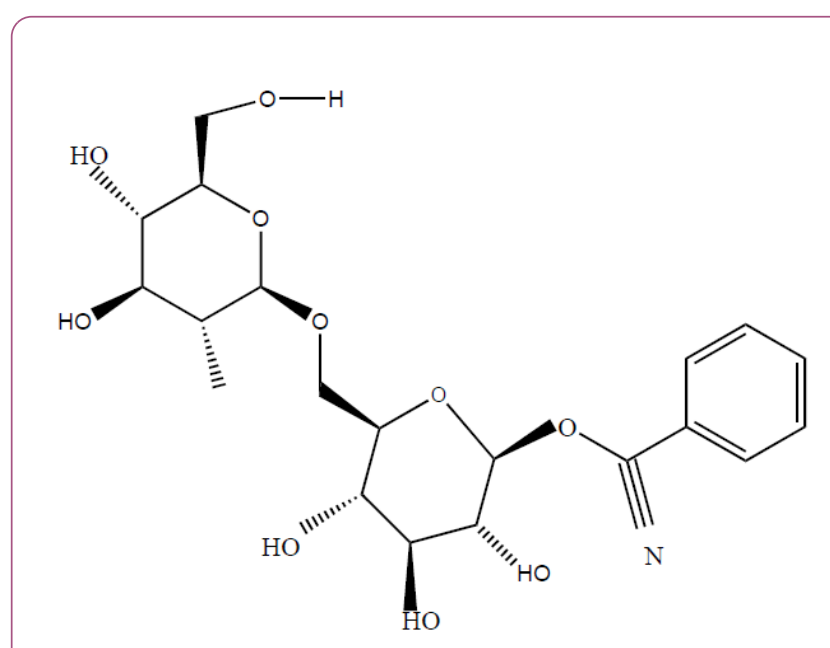

Figure 1 Structure of amygdalin.

\section{Antitumor effects of amygdalin}

Amygdalin is effectively used medicine by Chinese in recent 200 years by using bitter almond. Amygdalin was first found in bitter almond by Schrader in 1803. Amygdalin is one of the most used anticancer drugs in recent years as traditional treatment of cancer. It has many other names like vitamin B17, nitriloside and laetrile etc. Amygdalin was first discovered and purified by pair of chemist Robiquet and Boutron and they called it Liebig in 1837. It was used first time for cancer treatment by Russian doctor in 1845. Amygdalin was first used in America in 1820 for cancer. It is officially patented in $\mathbf{1 8 5 0 .}$ The analysis conducted by USA national cancer institute shows amygdalin which is given in form of oral or intravenous injection in Mexico do not fulfill the American drug production 
standards, regardless of all this Americans use it as drug for cancer treatment $[11,12]$.

To view this entire situation, National Cancer Institute conducted clinical studies of amygdalin. 6 patients out of 22 cases shows beneficial effects while other patients do not show positive effects. This study does not support efficiently antitumor effects of amygdalin. FDA of America proves the toxic effects of amygdalin in 1979. The import of amygdalin was stopped in America and Mexico during 1987. Amygdalin is listed as prescription drug in UK and can be used under doctor supervision as it produces cyanide which is toxic. Thus, as an antitumor drug it is produced in large quantity in Mexico and used also as drug. Many experimental results show that it has anti-cancerous activity. Some studies show that amygdalin can start apoptosis in human promyelocytic leukemia (HL-60) cells. Amygdalin repressed the production of human colon cancer SNU-C4 cell.

Amygdalin can start apoptosis by regulating expression of Bax and of Bcl-2 in prostate cancer DU145 and LNCaP cells. Amygdalin can reduce the existence of HeLa cells in rats. The endogenous mitochondrial pathway starts the apoptosis of HeLa cells. The detection results of human genome U133 microarray showed that 573 genes of HeLa cells had differential expression in amygdalin treated group compared with treated control group, JNK/c-jun pathway is involved in the process of amygdalin induced apoptosis in HeLa cells. However, the anti-cancerous activity of amygdalin is not completely understood.

\section{Results and Discussion}

The clinical trials shows that amygdalin had no steady anticancerous effect however it has an adverse effects such as headache and gastrointestinal tract reaction after large dose application [13]. The absence of amygdalin or laetrile's efficiency and adverse effects from cyanide poisoning led the FDA in USA and European commission ban it. However, it is available through internet because government cannot control these markets. Before given it to Cancer patients they should be informed about its adverse effects due to poisoning pf cyanogenic compounds after oral ingestion of laetrile. The danger could increase when amygdalin is taken with vitamin C. Some components of amygdalin may possess anti-neoplastic character which may be active against antitumor ingredient [14].

\section{Conclusion}

According to some studies amygdalin may play a secondary role in the cure of cancer. The pharmacological activity of amygdalin in diabetes, atherosclerosis, immune suppression, leprosy and other diseases is not clearly understood but however in some extent it is helpful to cure these diseases. There is need to investigate the mechanism of amygdalin and its pharmacological activity and produce anticancerous drug. Efforts in the future need to be put onward to expand pharmacokinetic profile, to inspect their metabolism, as well as to test their efficiency by oral and topical administration. In a few years these compounds are anticipated to overcome present difficulties and enter in clinical trials.

\section{References}

1. Holzbecher MD, Moss MA, Ellenberger HA (1984) The cyanide content of laetrile preparations, apricot, peach and apple seeds. J Toxicol Clin Toxico 22: 341-347.

2. Do JS, Hwang JK, Seo HJ, Woo WH, Nam SY (2006) Antiasthmatic activity and selective inhibition of type 2 helper $\mathrm{T}$ cell response by aqueous extract of semen armeniacae amarum. Immunopharmacol Immunotoxicol 28: 213-225.

3. Huaping Z, Liwen C, Wenbin L, Hanchu L (2004) Effect of amygdalin on the proliferation of hyperoxia-exposed type II alveolar epithelial cells isolated from premature rat. J Huazhong Univ Sci Technolog Med Sci 24: 223-225.

4. Perez JJ (2013) Amygdalin analogs for the treatment of psoriasis. Future Med Chem 5: 799-808.

5. Guo J, Wu W, Sheng M, Yang S, Tan J (2013) Amygdalin inhibits renal fibrosis in chronic kidney disease. Molecular Medicine Reports 7: 1453-1457.

6. Hwang HJ, Lee HJ, Kim CJ, Shim I, Hahm DH (2008) Inhibitory effect of amygdalin on lipopolysaccharide-inducible TNF-alpha and IL-1beta mRNA expression and carrageenan-induced rat arthritis. J Microbiol Biotechnol 18: 1641-1647.

7. Zhu YP, Su ZW, Li CH (1994) Analgesic effect and no physical dependence of amygdalin. Zhongguo Zhong Yao Za Zhi China J Chinese materia medica 19: 105-107.

8. Shim SM, Kwon H (2010) Metabolites of amygdalin under simulated human digestive fluids. Int J Food Sci Nutr 61: 770-779.

9. Heikkila RE, Cabbat FS (1980) The prevention of alloxan-induced diabetes by amygdalin. Life sci 27: 659-662.

10. Song Z, Xu X (2014) Advanced research on anti-tumor effects of amygdalin. J Cancer Res Ther 10: 3.

11. Chen Y, Ma J, Wang F, Hu J, Cui A, et al. (2013) Amygdalin induces apoptosis in human cervical cancer cell line HeLa cells. Immunopharmacol Immunotoxicol 35: 43-51.

12. Fukuda T, Ito $\mathrm{H}$, Mukainaka T, Tokuda $\mathrm{H}$, Nishino $\mathrm{H}$, et al. (2003) Anti-tumor promoting effect of glycosides from Prunus persica seeds. Biol Pharm Bull 26: 271-273.

13. Milazzo S, Lejeune S, Ernst E (2007) Laetrile for cancer: A systematic review of the clinical evidence. Support Care Cancer 15: 583-595.

14. O'brien B, Quigg C, Leong T (2005) Severe cyanide toxicity from 'vitamin supplements'. Eur J Emerg Med 12: 257-258. 